\title{
Multivalent vaccine formulation with BmVAL-I and BmALT-2 confer significant protection against challenge infections with Brugia malayi in mice and jirds
}

\author{
This article was published in the following Dove Press journal: \\ Research and Reports in Tropical Medicine \\ 23 March 2011 \\ Number of times this article has been viewed
}

\section{Ramaswamy \\ Kalyanasundaram \\ Padmavathi Balumuri \\ Department of Biomedical Sciences, College of Medicine, University of Illinois Rockford, IL, USA}

Purpose: Lymphatic filariasis, a mosquito-borne infection, affects 120 million people in 83 different countries. Mass drug administration is fully underway in several parts of the world to eradicate this infection by year 2020. Drugs alone are highly efficient treatments, but long-term sustainable prophylaxis requires an effective vaccine. No vaccines are available for humans and animals despite several potential candidate vaccine antigens having been identified. Brugia malayi vespid venom allergen homolog-like protein (BmVAL-1) and B. malayi abundant larval transcript (BmALT-2) are two of the most promising vaccine candidates. We evaluated various vaccination regimens consisting of DNA and protein antigens and evaluated the potential of monovalent and multivalent vaccine formulations in mice and jird animal models.

Methods: Mice and jirds were vaccinated with monovalent DNA preparations of BmVAL-1 or BmALT-2 in pVAX-1 vector or monovalent protein preparations of $\mathrm{rBmVAL}-1$ and rBmALT-2 in alum using a homologous or heterologous prime boost approach. These vaccine regimens were then compared with a multivalent vaccine formulation consisting of DNA or hybrid protein formulation of the two antigens. Challenge experiments were performed with B. malayi $\mathrm{L} 3$ in mice and jirds to evaluate the degree of protection, and immunological parameters were determined in mice and humans to elucidate the characteristics of the protective immune responses.

Results: Vaccination with monovalent BmVAL-1 vaccine conferred 39\% (DNA vaccine) to 54\% (DNA prime and protein boost) protection in mice. A similar degree of protection was observed in jirds (50\% to 52\%). Monovalent BmALT- 2 afforded 51\% to $75 \%$ protection in mice and 58\% to $79 \%$ protection in jirds. Our testing of a multivalent formulation of BmVAL-1 and BmALT-2, showed $57 \%$ to $82 \%$ protection in mice and $77 \%$ to $85 \%$ protection in jirds. A heterologous prime boost approach using the multivalent vaccine gave the highest degree of protection in both mice and jirds. Serological analysis in mice showed that BmVAL-1 vaccination induced an IgG1, IgG2a, and IgG3 antibody response, whereas BmALT-2 vaccination predominantly induced an IgG1 and IgG3 antibody response. Cytokine responses of antigen-responding cells in the spleen secreted predominantly IFN- $\gamma$ and IL-5 in response to BmVAL-1, and IL-4, and IL-5 in response to BmALT-2.

Conclusion: A multivalent vaccine formulation of BmVAL-1 and BmALT-2 given as a prime boost regimen gave significant protection against lymphatic filariasis caused by $B$. malayi in mice and jirds. Because putatively immune endemic normal subjects also carry protective antibodies against BmVAL-1 and BmALT-2, developing this multivalent formulation as a prophylactic vaccine against $B$. malayi for human and veterinary use has great potential.

Keywords: Brugia malayi, BmALT-2, BmVAL-1, vaccine, lymphatic filariasis
Professor and Head, Department of Biomedical Sciences, University of Illinois Rockford, College of Medicine, I60I Parkview Avenue, Rockford, IL 6I I07, USA

Tel +l 815 395-5696

$\mathrm{Fax}+\mathrm{I} 815$ 395-5684

Email ramswamy@uic.edu 


\section{Introduction}

Human lymphatic filariasis is a mosquito-borne disease caused mainly by two filarial parasites, Brugia malayi and Wuchereria bancrofti. The disease affects over 120 million people living in over 83 different countries in the tropical and subtropical regions of the world. ${ }^{1}$ Diethylcarbamazine (DEC) is an effective drug against this infection. ${ }^{2,3}$ Essentially, this drug clears the microfilaria stage of the parasite thereby preventing transmission. A combination of DEC with albendazole or ivermectin is currently used as a drug of choice for multidrug administration (MDA) strategy to control this infection in regions where it is endemic. ${ }^{3}$ The ultimate goal is to eliminate the disease by 2020. MDA strategy is proving to be very successful in several parts of the world. Despite its initial success, some endemic regions have already started facing significant difficulties in implementing the MDA program due to political unrest or lack of compliance. ${ }^{4-7}$ To be effective, MDA needs to be repeated annually for at least 5 years (WHO) ${ }^{8}$ Vector control measures are not as successful as treating the patient, to control the infection. However, since the vector population is very high in these regions, multiple treatments with MDA are necessary for consistent protection. MDA, especially albendazole as part of such a regimen raises concern because of the potential for drug resistance. In fact, resistance to the benzimidazole group of drugs has already been reported in the treatment of B. malayi and $W$. bancrofti ${ }^{9}$ Thus repeated administration of albendazole each year in the endemic region may not be ideal. A more practicable and sustainable approach to preventing this infection in a large population would be a prophylactic vaccination possibly with simultaneous mass screening and treating infected individuals with DEC or combination drugs such as that used in MDA.

Protective immunity against lymphatic filarial parasite has been demonstrated in both humans and animals. ${ }^{10,11}$ Certain individuals living in the endemic areas under the same environmental conditions as infected individuals do not show any symptoms of the disease, but carry a high titer of antibodies against the filarial parasites in their sera. Previous studies from our laboratory showed that these circulating antibodies can participate in the killing of infective third stage (L3) B. malayi larvae in vitro through an antibody dependent cell cytotoxicity (ADCC) mechanism. ${ }^{12}$ Similarly, animal studies have also shown that vaccination with irradiated third stage larvae (L3) of B. malayi confer significant protection against challenge infections. ${ }^{10}$ These findings provided strong evidence that protective immunity against $B$. malayi and $W$. bancrofti can be induced in human and animals.
However, identifying the host protective antigens and the development of a suitable vaccine against lymphatic filariasis has been severely hampered by the complicated life cycle of the parasite and the difficulty in maintaining $W$. bancrofti life cycle stages under laboratory conditions. Despite these difficulties, several potential candidate vaccine antigens have been reported from many laboratories. ${ }^{12-15}$ Completion of the B. malayi genome substantially boosted the vaccine antigen discovery.

Using a phage display-based iterative screening of a B. malayi L3 cDNA library with immune human sera, our laboratory previously showed that $B$. malayi vespid venom allergen homolog-like protein (BmVAL-1) and B. malayi abundant larval transcript-2 (BmALT-2) are potential vaccine candidates. ${ }^{14}$ Vaccine potential of both BmVAL-1 (BmVAL-1) and ALT-2 has already been reported previously by other groups. ${ }^{16-19}$ Thus the powerful phage display-based parasite cDNA expression library screening confirmed previous reports and narrowed down the candidate vaccine antigens to VAL-1 and ALT-2. VAL-1 belongs to a family of proteins called Ancylostoma caninum secreted proteins or ASP. ${ }^{20}$ VAL-1 homologs have been reported from Anclostoma duodenale, Necator americanus, Onchocerca volvulus, and Meloidogyne incognita. ${ }^{21-23}$ Although VAL-1 and ALT-2 are excellent vaccine candidates, their functional role in the parasite is not fully understood.

Conventional methods of immunization with recombinant proteins require co-administration with adjuvants, and usually have to be given in multiple doses. Moreover, a cold chain is needed to transport and store the protein vaccine. This requirement poses significant problems especially in tropical regions where the disease is highly prevalent. Immunization with DNA vaccine can overcome the need for such requirements associated with conventional protein immunization. In addition, DNA vaccines are easy to prepare and can be stored with relative ease, eliminating the need for a cold chain. More importantly, DNA-based vaccines can induce a high level of protective immune responses and elicit both cellular and humoral immune responses as has been demonstrated against several pathogens. ${ }^{24}$ Finally, multiple DNAs can easily be incorporated into the vaccine vector to generate a multivalent DNA vaccine, which is a great advantage over protein vaccine. A recent study by Anand et $\mathrm{al}^{31}$ showed that BmALT-2 and BmVAH when given as a cocktail vaccine can confer significant protection. In this manuscript we present data to evaluate the vaccine potential of a multivalent combination of BmVAL-1 and BmALT-2 as a potential DNA vaccine against lymphatic filariasis. 


\section{Materials and methods \\ Sera}

Sera samples used in this study were from archived samples stored at the Mahatma Gandhi Institute of Medical Sciences, Sevagram, India. These samples were collected as part of epidemiological surveys in and around Wardha, where lymphatic filariasis is endemic. Use of human subjects in this study and the protocols and the consent forms were approved by the Institutional Research Board of the University of Illinois, Rockford and Mahatma Gandhi Institute of Medical Sciences, Sevagram, India.

No demographic data were available for this study, except that the sera samples were classified into microfilaremic (MF), chronic pathology (CP), or endemic normals (EN) based on the detection of circulating parasites, parasite antigens, or evaluation of clinical symptoms of lymphatic filariasis. Circulating microfilariae were detected in the blood of subjects as described previously. ${ }^{12,14}$ The presence of circulating antigen was detected using an $\mathrm{Og} 4 \mathrm{C} 3 \mathrm{kit}$ and a WbSXP-based enzyme-linked immunosorbent assay (ELISA). Subjects with no circulating antigen or microfilariae were classified as EN, whereas subjects with circulating microfilariae and/ or circulating antigen, as detected by ELISA, were classed as MF. Subjects showing lymphedema and other visible clinical symptoms of filariasis were grouped into CP. Control nonendemic normal (NEN) sera were collected at the University of Illinois Clinic at Rockford, IL.

\section{Parasites}

Brugia malayi L3s were obtained from the NIAID/NIH

Filariasis Research Reagent Resource Center (FR3) at the University of Georgia, Athens, GA.

\section{Construction of monovalent and multivalent DNA vaccines}

To prepare monovalent vaccine, codon optimized BmVAL-1 (Acc: AF042088) or Bmalt-2 (Acc: U84723) genes were cloned into the eukaryotic expression vector pVAX1 (Invitrogen, Carlsbad, CA) using insert specific primers. ${ }^{14,25}$ To prepare multivalent vaccine, codon optimized BmVAL-1 gene was first cloned into pVAX1 vector with no stop codon using already published primer sequences with a pst I site. Codon optimized Bmalt-2 gene was then inserted into this clone using gene specific primers. PCR parameters for all the constructs were: $94^{\circ} \mathrm{C}$ denaturation for $30 \mathrm{~s}, 50^{\circ} \mathrm{C}$ primer annealing for $30 \mathrm{~s}$, and $72^{\circ} \mathrm{C}$ primer extension for $30 \mathrm{~s}$ for 30 cycles; a final extension of 5 minutes was performed at $72^{\circ} \mathrm{C}$. Insert DNA was finally sequenced to ensure authenticity of the cloned nucleotide sequence on both strands. Plasmids were maintained and propagated in E. coli Top 10F' cells. Plasmids were purified using an endotoxin free plasmid extraction kit (Qiagen, Valencia, CA). DNA was analyzed by agarose gel electrophoresis and quantified in a spectrophotometer (OD 260/280, ratio > 1.8).

\section{Expression and Purification of recombinant proteins}

Recombinant BmVAL-1 and rBmALT-2 were expressed in pRSET-A vector and purified using an immobilized cobalt metal affinity column chromatography as described previously from our laboratory. ${ }^{18,19}$ Endotoxins in the recombinant preparations were removed by passing the recombinant proteins through polymyxin B affinity columns (Thermo Fisher Scientific, Rockford, IL) and the levels of endotoxin in the final preparations were determined using an E-TOXATE kit (Sigma-Aldrich, St Louis, MO) as per manufacturer's instructions. Endotoxin levels in the final preparations $(0.005 \mathrm{EU} / \mathrm{mL})$ were below detection limits in these recombinant protein preparations.

\section{Immunoreactivity of the various human sera}

To determine whether the human sera samples carried antibodies against BmVAL-1 or BmALT-2, we performed an ELISA as described before. ${ }^{12,14}$ For isotype specific ELISA, alkaline phosphatase conjugated goat anti-human IgG1, anti-human IgG2, anti-human IgG3, and anti-human IgG4 antibodies (Sigma) were used as the secondary antibodies.

\section{Immunization protocol for mice and jirds}

Six-week old male Balb/c mice and 35-40 g outbred male Mongolian gerbils (jirds) purchased from Charles River Laboratories (Wilmington, MA) were used in these experiments. Animals were treated according to the guidelines in the Guide for the Care and Use of Laboratory Animals. Humane use of animals in this study and the protocol were approved by the IACUC committee at the College of Medicine, University of Illinois Rockford. The reason for using two different animal models to test the vaccine formulations is that the $B$. malayi parasite does not mature into adults in mice, so that vaccine-induced protection against the L3 stages can be evaluated in the mouse model. In addition, significant immunological parameters can be measured in mice. Conversely, B. malayi parasite develops into mature adult worms in jirds. Therefore, we can evaluate 
vaccine-induced protection against adult worm establishment in jirds.

The three sets of experiments were: 1) monovalent BmVAL-1 vaccination, 2) monovalent BmALT-2 vaccination, and 3) multivalent BmVAL-1/BmALT-2 vaccination. Each set of experiments had four groups a) DNA prime plus DNA boost (homologous), b) protein prime plus protein boost (homologous), c) DNA prime plus protein boost (heterologous) and pVAX plus alum controls. Each group consisted of 10 animals each. All animals were immunized subcutaneously with codon optimized DNA (100 $\mu \mathrm{g})$ in $50 \mu \mathrm{L}$ volume or with recombinant protein $(150 \mu \mathrm{g})$ plus alum in $50 \mu \mathrm{L}$ volume. Control group received $100 \mu \mathrm{g}$ of pVAX1 blank vector or $50 \mu \mathrm{L}$ of alum. Blood samples were collected at frequent intervals, and sera separated and stored at $-80^{\circ} \mathrm{C}$. The protocol followed for immunizing mice and jirds is schematically represented in Figure 1.

\section{Protection studies in mice}

Challenge studies were conducted in mice by surgically implanting 20 B. malayi live infective L3s into the peritoneal cavity in a micropore chamber as described previously. ${ }^{12,26}$ Aseptic conditions were followed for the surgical procedures; 48 hours after implantation, chambers were recovered from the peritoneal cavity and viability of the larvae was determined under a light microscope. The percentage of protection was expressed as the number of dead parasites/ number of total parasites recovered $\times 100$.

\section{Splenocyte proliferation and cytokine assays}

Single cell suspension of spleen cells $\left(0.5 \times 10^{6}\right.$ cells per well suspended in $200 \mu \mathrm{L}$ media) were prepared from each mouse and cultured in triplicate wells with either 1) $1 \mu \mathrm{g} / \mathrm{mL}$ rBmVAL-1, 2) $1 \mu \mathrm{g} / \mathrm{mL}$ rBmALT-2, 3) $1 \mu \mathrm{g} / \mathrm{mL}$ rBmVAL-1 + BmALT-2, 4) a non-specific recombinant protein $(1 \mu \mathrm{g} / \mathrm{mL}$ of Schistosoma mansoni G-binding protein), or 5) were left unstimulated in the media. All cells were incubated for 3 days

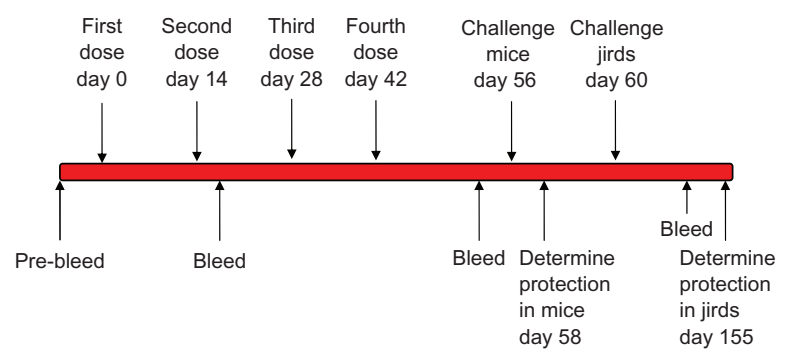

Figure I Schematic representation of the vaccination protocol used in mice and jirds. at $37^{\circ} \mathrm{C}$ with $5 \% \mathrm{CO}_{2}$. After 3 days, $3 \mathrm{H}$-thymidine $(0.5 \mathrm{lCi}$ per well, Amersham Biosciences) was added to each well and further incubated. Cells were harvested 16 hours later and 3 $\mathrm{H}$-thymidine uptake was measured in a liquid scintillation counter and expressed as stimulation index $(\mathrm{SI})=$ (counts per minute of stimulated cultures counts per minute of unstimulated cultures). Cell culture supernatants collected from the above spleen cultures were assayed for IFN- $\gamma$, IL-4, IL-5, and IL-10 using an ELISA kit purchased from eBioscience Inc. (San Diego, CA).

\section{BmVAL-I and BmALT-2 specific IgG antibodies in the sera of immunized mice}

Titer of anti-BmVAL-1 and anti-BmALT-2 specific antibodies was determined in the sera of immunized mice using an ELISA as described previously. ${ }^{12,14}$ Pre immune sera served as controls. Horseradish peroxide (HRP) conjugated goat anti-mouse IgG was used as the secondary antibody (Thermo Fisher Scientific) for mouse assay. O-Phenylenediamine dihydrochloride (OPD) (Sigma) was used as the substrate and optical density (OD) was measured at $405 \mathrm{~nm}$.

Anti-BmVAL-1 and anti-BmALT-2 specific IgG1, IgG2a, IgG2b, IgG3, and IgG4 antibodies were determined in the sera of mouse using a mouse antibody isotyping kit purchased from Thermo Fisher Scientific. All ELISAs were performed according to the manufacturer's recommendation and absorbance was read at $405 \mathrm{~nm}$. Respective HRP-labeled goat anti-IgG isotype antibody was used as the secondary antibodies and color was developed using OPD substrate.

\section{Challenge studies in jirds}

Jirds were challenged with 100 B. malayi L3s and worm establishment was determined on day 95 after challenge as described previously. ${ }^{27}$ Jirds are permissive hosts for $B$. malayi and the worms mature into adult males and females in about 75 days. Presence of mature worms in the control group of jirds was confirmed by demonstrating microfilariae in their blood on day 80 after challenge. Percent reduction in the worm establishment was calculated using the formula: average number of worms recovered from control worms - average number of worms recovered from vaccinated animals/average number of worms recovered from control animals $\times 100$.

\section{Statistical analysis}

Statistical analysis was performed using Sigmastat program (Jandel Scientific, San Rafel, California) and Statview (SAS Institute, Cary, NC) software. Wilcoxon signed rank test was used to compare paired data; comparison between the groups 
was performed using the Mann-Whitney $U$ test. $P$ value of $P<0.05$ was considered statistically significant.

\section{Results}

\section{EN individuals carry high titer of antibodies against BmVAL-I and BmALT-2}

Significant anti-BmVAL-1 and anti-BmALT-2 IgG antibodies were present in the sera of EN subjects compared with $\mathrm{MF}$ subjects $(P<0.01)$ and $C P$ subjects $(P<0.005)$ (Figure $2 \mathrm{~A})$. NEN subjects did not carry IgG antibodies against both the antigens. Subsequent analysis of the IgG isotype of antibodies in the sera of EN subjects showed that antiBmVAL-1 and antiBmALT-2 antibodies were predominantly of $\operatorname{IgG} 1$ and IgG3 isotypes (Figure 2B).

\section{High titer of antibody responses in the sera of immunized mice}

Previous studies from our laboratory showed that mice vaccinated with $B$. malayi antigens elicited significant host protective IgG antibodies. ${ }^{12,14,25}$ Therefore, in this study, we focused mainly on measuring $\operatorname{IgG}$ antibody titers in the sera of immunized mice. Monovalent immunization with

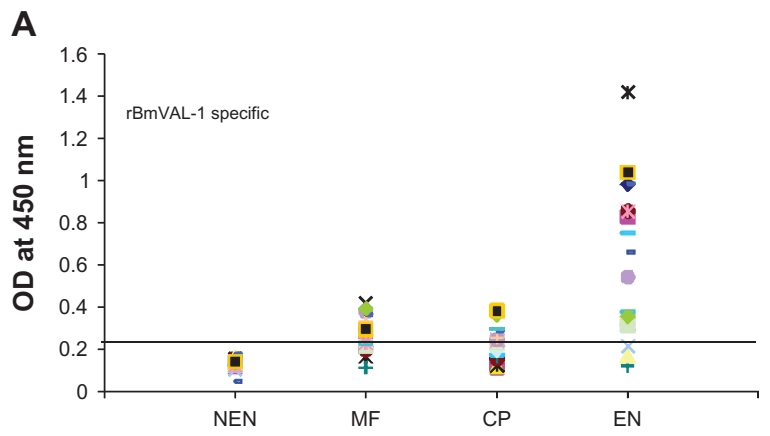

B

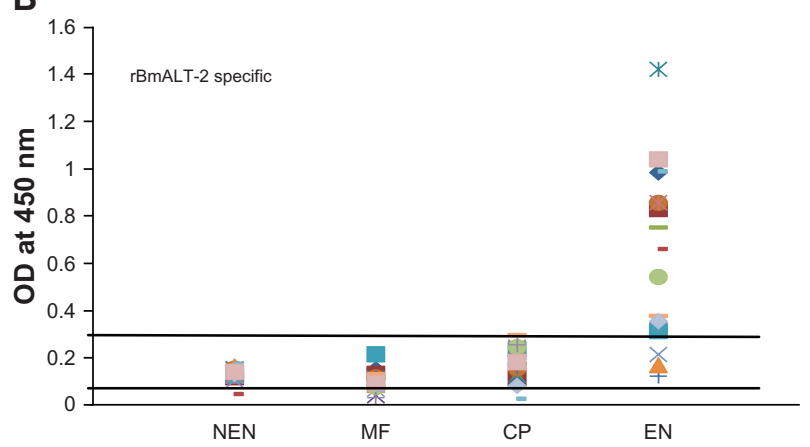

Figure 2A Titer of BmVAL-I specific $\lg G$ antibodies $\mathbf{A}$ ) and BmALT-2 specific $\lg G$ antibodies B) in the sera of human subjects. Titer of antigen specific $\lg G$ antibodies were determined in sera samples from microfilaremic $(n=20)$, chronic pathology $(n=20)$ and endemic normal (NEN) $(n=20)$ subjects using an ELISA. Sera samples from NEN subjects served as controls. Each spot represents sera samples from one individual. The cut off value (mean OD \pm 3 times SD of NEN sera) is indicated by a line drawn parallel to $x$-axis.
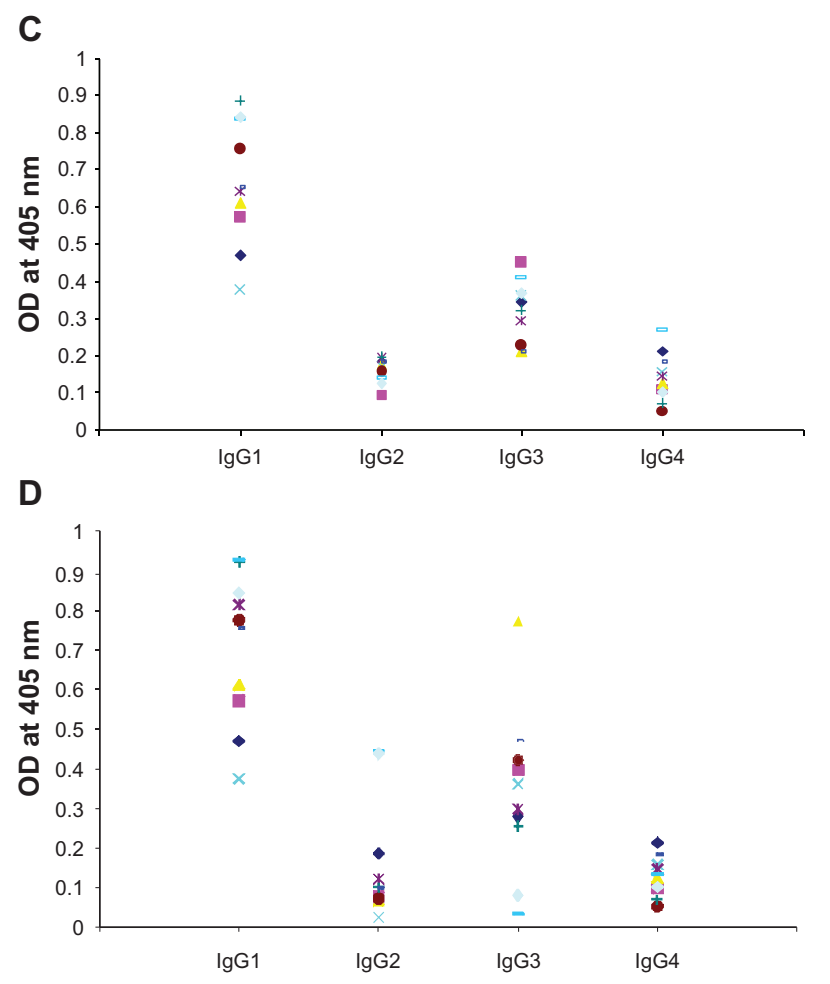

Figure 2B Titer of $\lg G$ isotype specific antibodies against $r B m V A L-I \quad C)$ and rBmALT-2 D) in the sera samples of endemic normal subjects. Values were determined by specific ELISA for each isotype. Each spot represent sera samples from one individual. The IgG isotype antibody patterns against BmVAL-I and BmALT-2 were comparable in the sera samples; $\mathrm{N}=20$.

BmVAL-1 (Figure 3A) and monovalent immunization with BmAlt-2 (Figure 3B) both elicited significant $(P<0.005)$ titers of anti-BmVAL-1 and anti-BmALT-2 IgG antibodies in the sera of mice. Compared with controls, the prime boost immunized group gave the maximum titer of antibodies followed by protein immunized and DNA immunized groups (Figure 3). Immunization with the multivalent vaccine formulation (BmVAL-1 $+B m A L T-2)$ also elicited significant IgG antibody titers against both rBmVAL-1 and rBmALT-2 (Figure $3 \mathrm{C}$ ) and the titers were comparable, suggesting that the antigens do not interfere with each other or compete for dominance. An interesting finding was that the multivalent vaccine elicited significantly higher $(P<0.001)$ titer of IgG antibodies in mice compared with any of the monovalent vaccines (Figure 3 ). These findings suggested that the two antigens in the multivalent formulation synergistically increase the vaccine-induced antibody responses.

Overall, protein vaccination elicited higher titer of IgG antibodies compared with DNA vaccines suggesting that protein vaccinations are highly immunogenic (Figure 3). This may be partially due to the adjuvant used along with the protein vaccine. Another observation was that a heterologous prime boost approach gave 
A
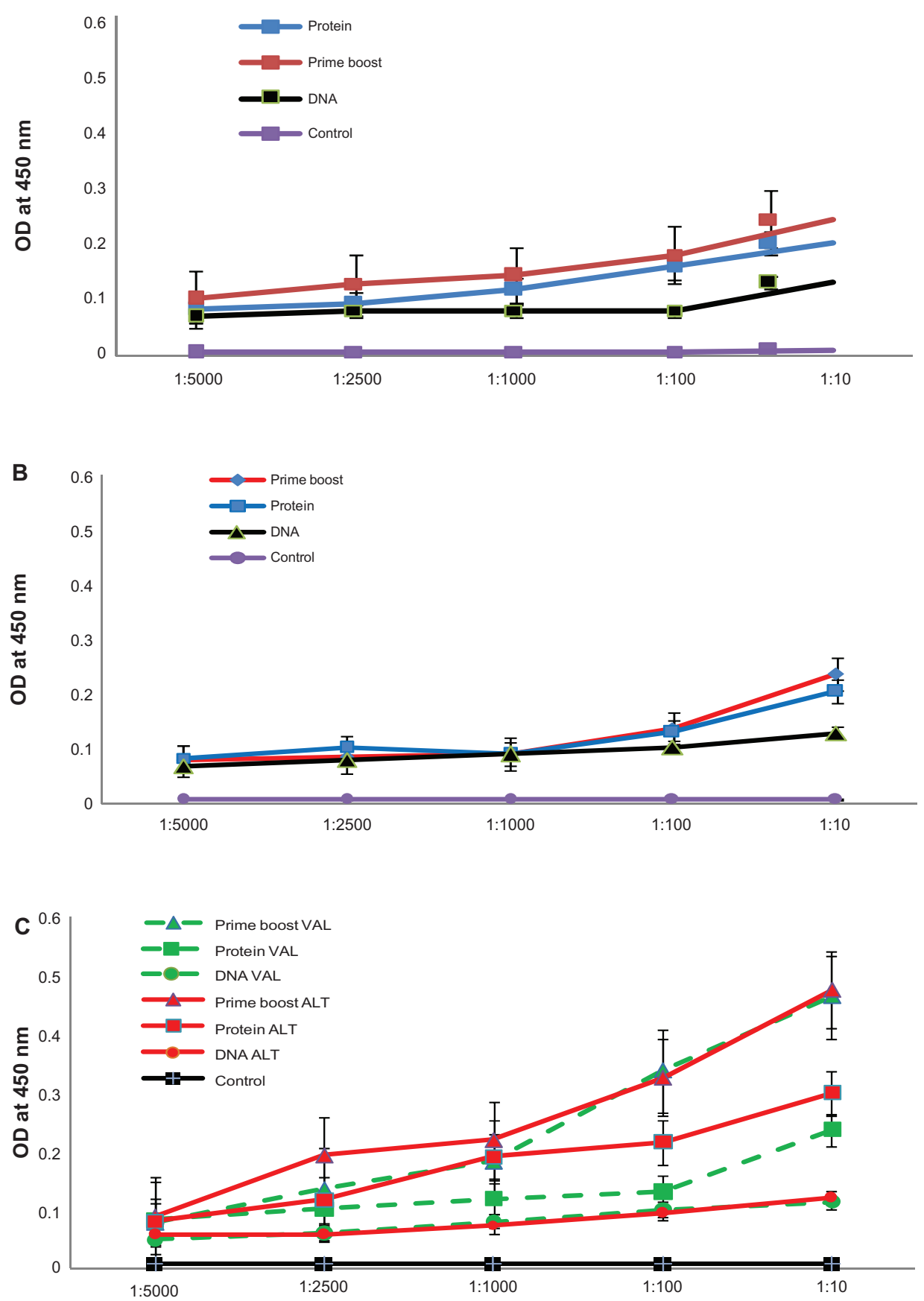

Figure 3 Titer of anti-VAL-I IgG and anti-ALT-2 $\lg G$ antibodies in the sera of immunized mice after 4 immunizations at 2-week intervals. lgG levels were measured using an ELISA. DNA - DNA vaccinated group, Protein - Protein vaccinated group, Prime boost - DNA Prime protein boost vaccinated group, and Control - vector plus alum controls. A) Sera collected from mice immunized with monovalent BmVAL-I vaccine. B) Sera collected from mice immunized with monovalent BmALT-2 vaccine. C) Sera collected from mice immunized with BmVAL-I/BmALT-2 multivalent vaccine. Dotted lines: values for anti-BmVAL-I lgG; solid lines: values for anti-BmALT-2 lgG; $N=10$. Data represent results from one of two experiments with comparable results.

a higher seroconversion than a homologous prime boost approach (Figure 3). Thus, in general, a heterologous prime boost approach appeared to stimulate the highest titer of antibodies.

IgG antibody subset analysis showed that BmVAL-1 vaccination elicited primarily $\operatorname{IgG} 1$ and $\operatorname{IgG} 2 \mathrm{a}$ isotype of antibodies, whereas, BmALT-2 vaccination induced $\operatorname{IgG1}$, IgG2a, and IgG3 isotype of antigen-specific antibody responses (Figure 4). Antigen-specific IgG4 antibody response was not evident. The prime boost approach significantly amplified the IgG isotype responses. After a multivalent vaccination regimen of $\operatorname{IgG} 1, \operatorname{IgG} 2 \mathrm{a}$, and 


\section{A}

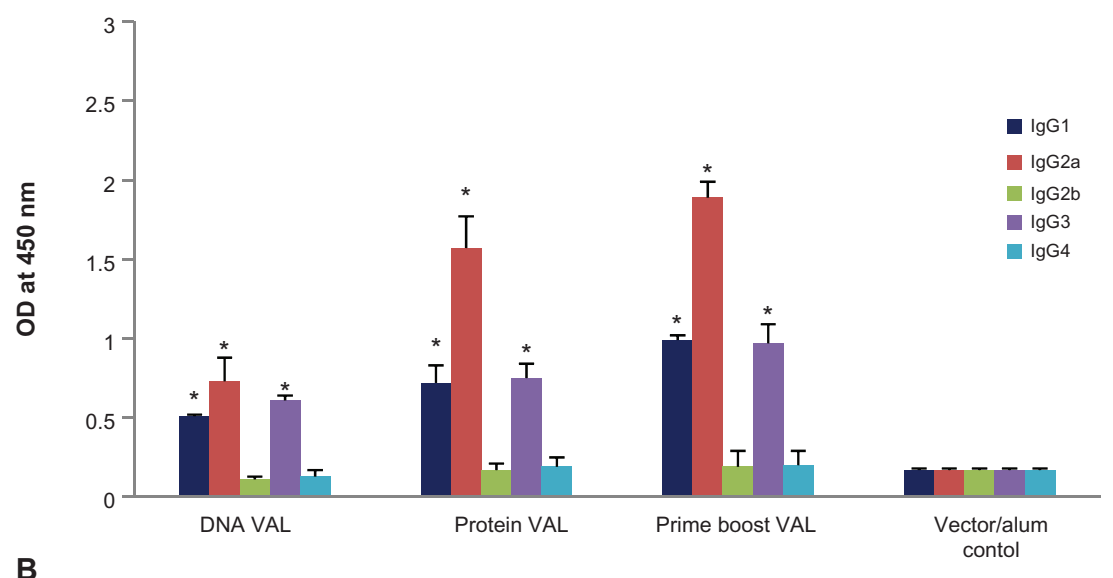

B
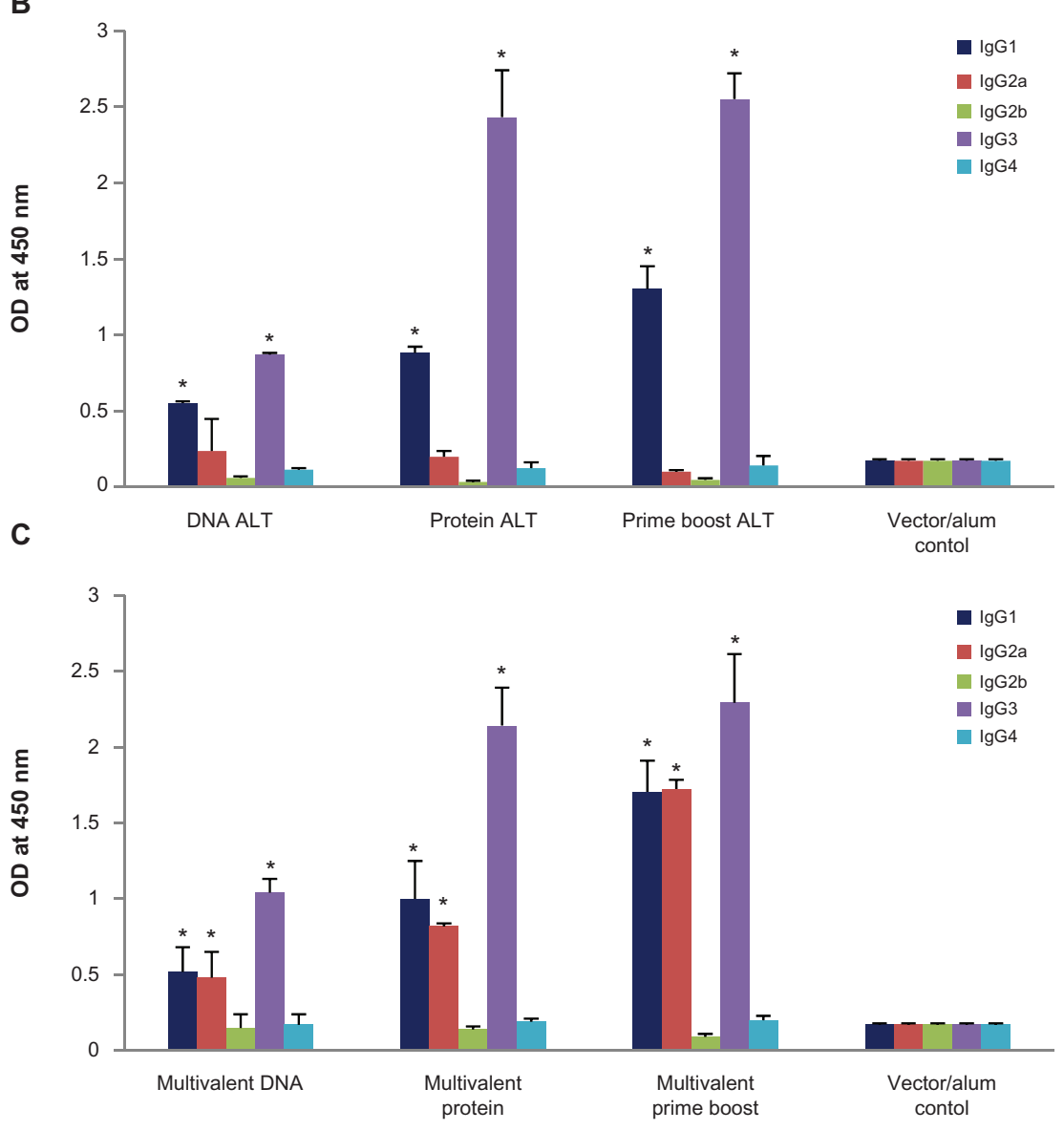

Figure 4 Levels of antiVAL-I and anti-ALT-2 IgG isotype of antibodies were measured in the sera of immunized mice 2 weeks after the last immunization. A) Monovalent BmVAL-I vaccinated mice, B) Monovalent BmALT-2 vaccinated mice, and C) multivalent vaccinated mice; $\mathrm{N}=10$.

Note: *Significant $P<0.01$ compared with vector plus alum control group.

IgG3 subset of antigen, specific antibodies were present in the sera of mouse (Figure 4).

\section{Antigen-specific responses in the spleen of mice}

Spleen cells from immunized mice stimulated with either rBmVAL-1 or rBmALT-2 proliferated significantly (SI $10.8 \pm$
1.1 and SI $14.6 \pm 1.2$ respectively) compared with the media control (SI $2.1 \pm 0.9$ ). Spleen cells from mice immunized with the multivalent construct responded to both rBmVAL-1 (SI $18.9 \pm 2.6$ ) and rBmALT-2 (SI $23.5 \pm 3.1$ ) suggesting that a strong recall cellular response is generated to both BmVAL-1 and BmALT-2 following vaccination with the multivalent construct. 


\section{Cytokine analysis from proliferated culture supernatants}

To identify the cytokine profile of the antigen-responding cells, we collected the culture supernatant of mouse spleen cells stimulated with respective antigen (rBmVAL-1 or rBmALT-2) and measured the level of IFN- $\gamma$, IL-4, IL-5, and IL-10. These results showed that significant levels of IL-5 and IFN- $\gamma$ are secreted by the spleen cells in response to rBmVAL-1. Spleen cells stimulated with rBmALT-2 predominantly secreted IL-4 and IL-5 (Figure 5).

\section{Multivalent vaccine induces significant protection in mice and jirds}

The above results show that significant IgG antibodies are elicited following vaccination with monovalent and multivalent vaccine preparations. To test whether the

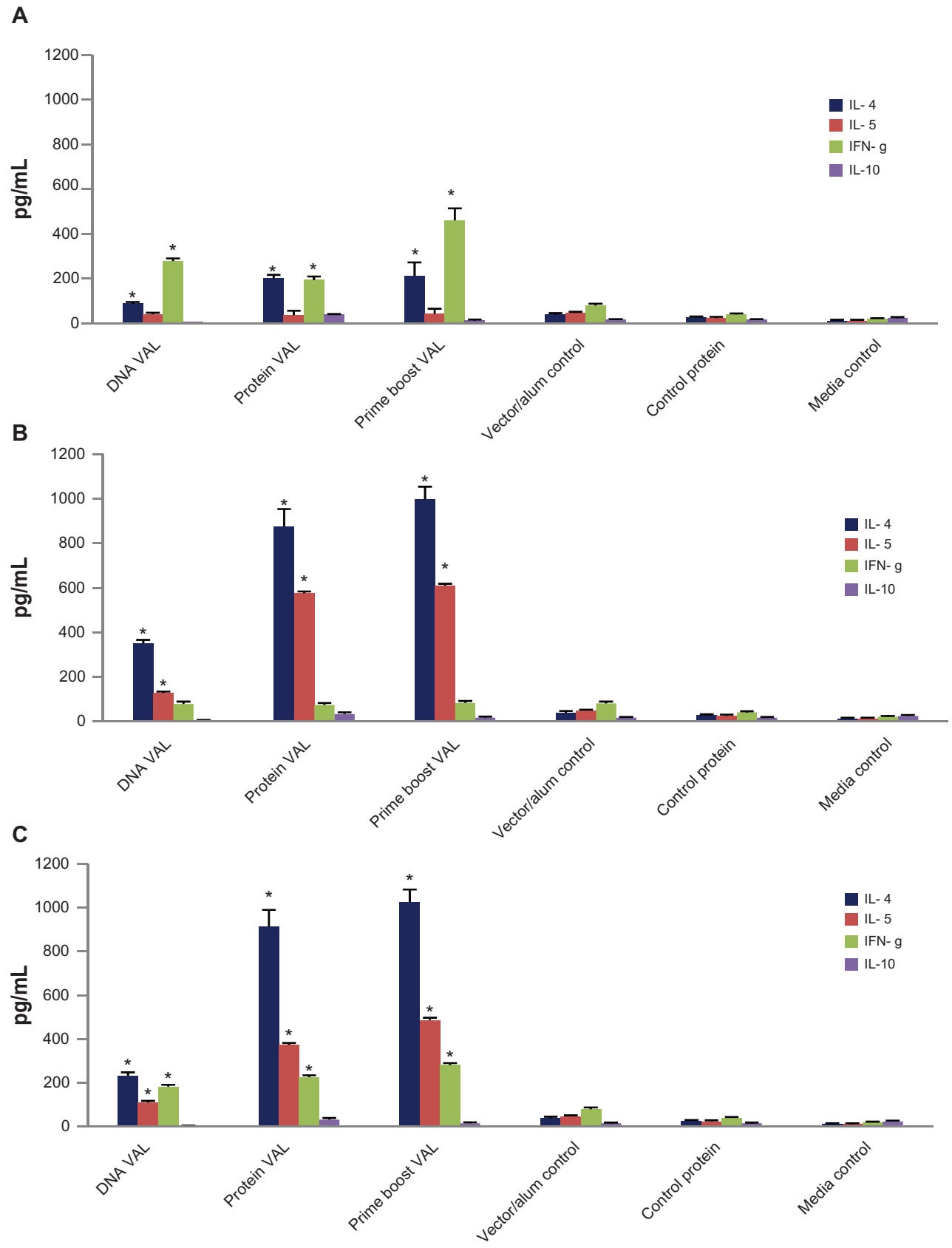

Figure 5 Cytokine responses in the culture supernatants of spleen cells stimulated for 72 hours with rBmVAL-I (A) rBmALT-2 (B), or rBmVAL-I-BmALT-2 hybrid protein (C) were measured using an ELISA. Concentrations of each cytokines are represented as $\mathrm{pg} / \mathrm{mL}$; spleen cells from vector/alum injected mice served as control. Additional controls include cells incubated in media alone and cells stimulated with a non-specific recombinant protein. Spleen cells from the prime boost group were used for the last two control groups; $\mathrm{N}=10$.

Note: *Significance $P<0.0$ I compared with control cells. 
immune responses elicited after vaccination are protective, we challenged vaccinated animals with live third stage infective larvae (L3) of B. malayi. Because the parasites do not reach maturity in mice, we used a standard micropore chamber challenge method. ${ }^{14}$ These studies showed that $39 \%$ to $74 \%$ protection can be achieved in mice after immunization with monovalent vaccine (Table 1). Protein vaccination gave better results than DNA vaccination. The prime boost regimen gave the best results overall. Vaccination with BmALT-2 gave a higher percent of protection compared with BmVAL-1. Similarly, a multivalent vaccination regimen gave $57 \%$ to $82 \%$ protection compared with the monovalent vaccination regimen. These findings suggested that BmVAL-1 and BmALT-2 synergistically enhance the protective immune responses in vaccinated animals when given as a multivalent vaccine (Table 1).

Analysis of the thick blood smear prepared from the control group of jirds on day 80 after challenge showed that all five jirds were positive for microfilaria, whereas microfilaria were not detected in the peripheral blood of vaccinated jirds (data not shown). Fifteen (15) days later we sacrificed the animals and counted the male and female worms in the peritoneal, pelvic, and pleural cavities and compared the results between controls and vaccinated groups (Table 2). Findings from vaccination of jirds also confirmed that the multivalent prime boost regimen gave the highest

Table I Percent larval death (protection) in mice challenged with 20 B. malayi L3

\begin{tabular}{|c|c|c|}
\hline Vaccination groups & $\begin{array}{l}\text { Mean } \pm \text { SD } \\
\text { live L3s }\end{array}$ & $\begin{array}{l}\text { Percent } \\
\text { protection }\end{array}$ \\
\hline $\begin{array}{l}\text { pVAXBmVAL-I DNA monovalent } \\
\text { homologous }\end{array}$ & $12.2 \pm 4.5$ & $39.0 \% \pm 1.7 \% *$ \\
\hline $\begin{array}{l}\text { rBmVAL-I protein monovalent } \\
\text { homologous }\end{array}$ & $10.4 \pm 3.1$ & $48.0 \% \pm 2.1 \% *$ \\
\hline $\begin{array}{l}\text { pVAXBmVAL-I DNA plus rBmVAL-I } \\
\text { monovalent heterologous }\end{array}$ & $9.2 \pm 2.2$ & $54.0 \% \pm 3.1 \% *$ \\
\hline $\begin{array}{l}\text { pVAXBmALT-2 DNA monovalent } \\
\text { homologous }\end{array}$ & $9.8 \pm 2.1$ & $51.0 \% \pm 2.5 \% *$ \\
\hline $\begin{array}{l}\text { rBmALT-2 protein monovalent } \\
\text { homologous }\end{array}$ & $7.0 \pm 1.1$ & $65.0 \% \pm 4.2 \% *$ \\
\hline $\begin{array}{l}\text { pVAXBmALT-2 DNA plus rBmALT-2 } \\
\text { monovalent heterologous }\end{array}$ & $5.1 \pm 0.5$ & $74.5 \% \pm 3.1 \% *$ \\
\hline $\begin{array}{l}\text { pVAXBmVAL-I/ALT-2 DNA multivalent } \\
\text { homologous }\end{array}$ & $8.6 \pm 0.1$ & $57.0 \% \pm 2.2 \% *$ \\
\hline $\begin{array}{l}\text { rBmVAL-I/rBmALT-2 protein } \\
\text { multivalent homologous }\end{array}$ & $5.2 \pm 1.1$ & $74.0 \% \pm 3.3 \% *$ \\
\hline $\begin{array}{l}\text { pVAXBmVAL-I/BmALT-2 DNA plus } \\
\text { rBmVAL-I/rBmALT-2 multivalent } \\
\text { heterologous }\end{array}$ & $4.4 \pm 0.4$ & $82.0 \% \pm 2.2 \% *$ \\
\hline pVAX + alum control & $20 \pm 0$ & $0 \%$ \\
\hline
\end{tabular}

Notes: Significance $* P<0.01$; $* * P<0.05$ compared with control.
Table 2 Percent worm reduction (protection) in jirds challenged with 100 B. malayi L3

\begin{tabular}{ll}
\hline Vaccination groups & $\begin{array}{l}\text { Percent } \\
\text { protection }\end{array}$ \\
\hline pVAXBmVAL-I DNA monovalent homologous & $50 \pm 3.7 \%$ \\
rBmVAL-I protein monovalent homologous & $40.0 \pm 3.1 \%$ \\
pVAXBmVAL-I DNA plus rBmVAL-I monovalent & $52.4 \pm 2.5 \%$ \\
heterologous & \\
pVAXBmALT-2 DNA monovalent homologous & $58.3 \pm 2.1 \%$ \\
rBmALT-2 protein monovalent homologous & $72.0 \pm 5.5 \%$ \\
pVAXBmALT-2 DNA plus rBmALT-2 monovalent & $78.5 \pm 3.2 \%$ \\
heterologous & \\
pVAXBmVAL-I/ALT-2 DNA multivalent homologous & $77.1 \pm 2.0 \%$ \\
rBmVAL-I/rBmALT-2 protein multivalent homologous & $79.9 \pm 3.5 \%$ \\
pVAXBmVAL-I/BmALT-2 DNA plus rBmVAL-I/rBmALT-2 & $85.0 \pm 1.4 \%$ \\
multivalent heterologous & \\
pVAX + Alum control & $0 \%$ \\
\hline
\end{tabular}

Note: Significance $P<0.01$ compared with control. Values were calculated as the percent reduction in worm establishment compared with control jirds that had a total of $39 \pm 7$ worms established.

rate of protection. No female worms were recovered from the multivalent vaccinated animals.

\section{Discussion}

Developing a vaccine against lymphatic filariasis has been challenging due to the complex life cycle of the parasite and the difficulty of obtaining enough parasite materials for research, especially of $W$. bancrofti. Our laboratory recently developed a phage expression library of different stages of W. bancrofti and B. malayi. ${ }^{14}$ Extensive screening of this library with sera from immune individuals yielded several potential candidate antigens. Many of these antigens show significant sequence identity and cross reactivity between $W$. bancrofti and B. malayi. In this study we analyzed two of the leading vaccine candidates, BmVAL-1 and BmALT-2. Both these antigens were significantly recognized by all EN sera and thus are potential vaccine candidates. Results presented in this study show that a multivalent vaccine formulation that combines these two antigens is much more immunogenic than a monovalent vaccine regimen of each antigen separately. Similarly, a heterologous prime boost approach using a DNA prime and protein boost gave significantly higher protection compared with a homologous prime boost approach.

Results from our studies show that immunization of mice with BmVAL-1 and BmALT-2 as a multivalent prime boost regimen conferred $82 \%$ protection. Since the worms do not mature into adult worms in mice a micropore chamber method was used to evaluate protection. This method of 
evaluating protection against B. malayi in mice is well established. ${ }^{25,26,28}$ The parasites develop into mature adults in jirds. Thus, parasite establishment can be evaluated as a determinant of protection in jirds. Vaccination in jirds also confirmed our results in mice. Multivalent vaccination using a prime boost regimen in jirds also confirmed that almost $85 \%$ protection can be achieved. While sterile immunity will be ideal, it is often very difficult to achieve that level of protection with a prophylactic vaccine for any parasitic infection. Nevertheless, close to $85 \%$ protection should substantially reduce the incidence of the infection at a much faster rate than multiple application of MDA alone, when combined with treatment of the infected individuals.

The vaccine candidates BmVAL- 1 and BmALT- 2 are the highly expressed proteins in the third stage larvae of B. malayi. ${ }^{29}$ Because of their abundance, these proteins are easily available to the host immune system. Previous immunization studies using either of these proteins show that vaccination with each one can confer significant protection in mouse and jird models. ${ }^{17-19,25}$

B. malayi vespid venom allergen homolog-like protein (BmVAH or BmVAL-1) or ASP, or sperm coating proteinlike protein (SCP), are part of the SCP family, which contain highly immunogenic proteins, which are expressed by a number of nematode parasites. ${ }^{14,19-21,30,31}$ Among these, ASP has been extensively evaluated for its vaccine potential. $^{20,21}$ Vaccination studies using BmVAL-1 in the lymphatic filariasis model show that significant protection can be achieved in mice against challenge infections. ${ }^{19,31}$ Although the true function of filarial VAL-1 is not fully understood, VAL-1 homologs are believed to be involved in the establishment of infection and transition to parasitism, as they are very abundant in the excretory-secretory (ES) material of infective larvae. ${ }^{20}$ Onchocerca volvulus ASP has angiogenic properties ${ }^{23}$ suggesting that VAL-1 may have a potential role in the pathogenesis. VAL-1 also has significant sequence homology with venom allergens from vespid wasps (Ag5) and fire ants (Ag3). ${ }^{30}$ A study by Visser et al $^{32}$ showed that VAL-1 and its homolog may have a role in the reproductive development of the male Ostertagia worms. Thus the VAL-1 homolog appears to be important for the establishment and survival of the parasite in the host ${ }^{20}$ and may have a possible role in immune evasion. ${ }^{29}$ Based on the reports to date, BmVAL-1 appears to be an ideal candidate for vaccine development against lymphatic filariasis.

Another vaccine candidate used in this study is BmALT-2. BmALT-2 and the closely related BmALT-1 together represent about $5 \%$ of the total $B$. malayi $\mathrm{L} 3 \mathrm{cDNA}$ indicating that they are the most abundant proteins in this parasite. ${ }^{17}$ BmALT-1 and BmALT-2 are also abundant in the protein secretions of the infective L3. ${ }^{33}$ Immunization with BmALT-1 or BmALT-2 can confer approximately $75 \%-76 \%$ reduction in worm load. ${ }^{17,18}$ This is probably the only filarial recombinant antigen that can afford this high protection rate. ${ }^{17}$ The mechanism of this high protection is not known, but research has shown that these proteins may play a significant role in host immunomodulation. ${ }^{29}$ Significant amounts of BmALT proteins are shown to be released from the glandular stockpiles of L3 larvae. Similar to BmVAL-1 vaccination results, our results with BmALT-2 vaccination also showed that a BmALT-2 heterologous vaccination regimen provides greater protection than monovalent or homologous regimens.

Since both BmVAL-1 and BmALT-2 conferred significant protection, we combined the two antigens as a multivalent formulation and compared the homologous and heterologous vaccine regimens. These studies showed that a multivalent heterologous vaccine regimen using BmVAL-1/BmALT-2 DNA to prime and BmVAL-1/BmALT-2 protein to boost gave the best protection results. Our findings also showed that combining BmVAL-1 with BmALT-2 can synergistically increase the protection efficiency of both the antigens. After multivalent vaccination, immunized mice developed a comparable titer of antigen-specific antibodies against both the antigens suggesting that BmVAL-1 and BmALT-2 do not interfere in the immunogenicity of the antigens. Thus the multivalent combination of BmVAL- 1 and BmALT-2 is an excellent choice for further vaccine development.

A major contributor to this synergistic effect of these two antigens may be that both the antigens selectively promoted IgG1 and IgG3 antibody responses in EN subjects although the antibody responses in mouse appear to be largely $\operatorname{IgG} 1$, IgG2a, and IgG3. Human IgG1 and IgG3 are cytophilic antibodies and can fix complement. Both IgG1 and IgG3 can also bind to Fc $\gamma R$ I on the surface of various effector cells which, when activated, can potentially kill B. malayi larvae..$^{12,14,34,35}$ Our previous studies show that antibodies in the sera of endemic normal individuals can participate in the killing of B. malayi $\mathrm{L} 3$ through an antibody dependent cell cytotoxicity (ADCC) mechanism. ${ }^{12,25}$ Thus the antigen-specific IgG1 and IgG3 subtype of antibodies that we observed against both BmVAL-1 and BmALT-2 in the sera of putatively immune EN subjects may have some functional significance. Mouse studies show that IgG1, IgG2a, and IgG3 antibody isotypes were increased following vaccination. The human counterparts of murine IgG subclasses are based on similarities 
in biological and functional activities. Murine $\operatorname{IgG} 2 \mathrm{a}$ and IgG2b and human IgG1 and IgG3 share the ability to fix complement and bind to protein antigens, whereas, murine IgG1 and human IgG4 are considered to be similar because of their property of binding to mast cells. Murine IgG3 and human IgG2 both recognize predominantly carbohydrate epitopes. All mouse subtypes can participate in ADCC. ${ }^{36}$ Mouse IgG3 and IgG2a immune complexes can bind strongly to Fc $\gamma \mathrm{RI}$ and trigger receptor mediated responses ${ }^{37}$ suggesting that these two isotypes may play a significant role in the vaccine-induced protection against B. malayi. Mouse IgG3 however is an early effector molecule of the immune system and appears early in the immune responses independently of $\mathrm{T}$ cell help. Thus, all three subtypes of $\mathrm{IgG}$ antibodies that we observed in vaccinated animals may have a significant role in the vaccine-induced protection in mice.

BmVAL-1 and BmALT-2 antigen-specific cells were present among the spleen cell population of the immunized animals as demonstrated by the recall responses. These cells in response to the antigens secreted IL-4, IL-5, and IFN- $\gamma$. Thus there was a mixed Th- $1 /$ Th2 response elicited in animals vaccinated with heterologous multivalent vaccine. Predominantly, the IFN $-\gamma$ response was a reaction to BmVAL-1, whereas BmALT-2 largely induced an IL-4/IL-5 response. In the murine system, gamma interferon produced by Th1 cells induces IgG2a and IgG3 in vitro; ${ }^{38} \mathrm{IL}-4$ produced by $\mathrm{Th} 2$ selectively stimulates $\mathrm{IgG} 1$ and $\operatorname{IgE}$.

In conclusion, results presented in this study show that a multivalent combination of BmVAL- 1 and BmALT-2 is an excellent vaccine for lymphatic filariasis caused by $B$. malayi. Since putatively immune EN subjects also carry IgG1 and IgG3 antibodies against BmVAL-1 and BmALT-2, developing this multivalent formulation as a prophylactic vaccine against B. malayi for human and veterinary use has great potential.

\section{Acknowledgments}

This study was supported by NIH grant (AI06474). B. malayi L3s were obtained from the NIAID/NIH Filariasis Research Reagent Resource Center (FR3) at the University of Georgia, Athens, GA. We would like to express our sincere thanks to Dr Maryada Venkata Reddy, Department of Biochemistry, Mahatma Gandhi Institute of Medical Sciences, Sevagram, Maharashtra, India, for providing human sera samples for this study.

\section{Disclosure}

The authors report no conflicts of interest.

\section{References}

1. Ottessen EA, Ramachandran C. Lymphatic filariasis, infection and disease: control strategies. Parasitol Today. 1995;11:129-131.

2. Weil GJ, Kastens W, Susapu M, et al. The impact of repeated rounds of mass drug administration with diethylcarbamazine plus albendazole on bancroftian filariasis in Papua New Guinea. PLoS Negl Trop Dis. 2008;2:e344.

3. Sudomo M, Chayabejara S, Duong S, Hernandez L, Wu WP, Bergquist R. Elimination of lymphatic filariasis in Southeast Asia. Adv Parasitol. 2010;72:205-233.

4. Parker M, Allen T. Does mass drug administration for the integrated treatment of neglected tropical diseases really work? Assessing evidence for the control of schistosomiasis and soil-transmitted helminths in Uganda. Health Res Policy Syst. 2011;9:3.

5. Bockarie MJ, Deb RM. Elimination of lymphatic filariasis: do we have the drugs to complete the job? Curr Opin Infect Dis. 2010;23: 617-620.

6. Bhullar N, Maikere J. Challenges in mass drug administration for treating lymphatic filariasis in Papua, Indonesia. Parasit Vectors. 2010;3:70.

7. Cantey PT, Rout J, Rao G, Williamson J, Fox LM. Increasing compliance with mass drug administration programs for lymphatic filariasis in India through education and lymphedema management programs. PLoS Negl Trop Dis. 2010;4:e728.

8. World Health Organization. Global Programme to Eliminate Lymphatic Filariasis. Available at: http://whqlibdoc.who.int/ publications/2010/9789241500722_eng.pdf. Accessed March 11, 2011.

9. Hoti SL, Dhamodharan R, Subramaniyan K, Das PK. An allele specific PCR assay for screening for drug resistance among Wuchereria bancrofti populations in India. Indian J Med Res. 2009;130:193-199.

10. Yate JA, Higash GI. Brugia malayi: vaccination with cobalt - attenuated infective stage larvae protects jird against homologous challenge. Am J Trop Med Hyg. 1985;34:1132-1137.

11. Michael E, Bundy DA. Herd immunity to filarial infection is a function of vector biting rate. Proc Biol Sci. 1998;265:855-860.

12. Veerapathran A, Dakshinamoorthy G, Gnanasekar M, Reddy MV, Kalyanasundaram R. Evaluation of Wuchereria bancrofti GST as a vaccine candidate for lymphatic filariasis. PLoS Negl Trop Dis. 2009; 3:e457.

13. Li BW, Rush AC, Mitreva M, et al. Transcriptomes and pathways associated with infectivity, survival and immunogenicity in Brugia malayi L3. BMC Genomics. 2009; 15:267.

14. Gnanasekar M, Rao KV, He YX, et al. Novel phage display-based subtractive screening to identify vaccine candidates of Brugia malayi. Infect Immun. 2004;72:4707-4715.

15. Selkirk ME, Rutherford PJ, Denham D, Partono F, Maizels RM. Cloned antigen genes of Brugia filarial parasites. Biochem Soc Symp. 1987;53:91-102.

16. Murray J, Gregory WF, Gomez-Escobar N, Atmadja AK, Maizels RM. Expression and immune recognition of Brugia malayi VAL-1, a homologue of vespid venom allergens and Ancylostoma secreted proteins. Mol Biochem Parasitol. 2001;118:89-96.

17. Gregory WF, Atmadja AK, Allen JE, Maizels RM. The abundant larval transcript-1 and -2 genes of Brugia malayi encode stage-specific candidate vaccine antigens for filariasis. Infect Immun. 2000;68: 4174-4179.

18. Thirugnanam S, Pandiaraja P, Ramaswamy K, et al. Brugia malayi: comparison of protective immune responses induced by Bm-alt-2 DNA, recombinant Bm-ALT-2 protein and prime-boost vaccine regimens in a jird model. Exp Parasitol. 2007;116:483-491.

19. Anand SB, Gnanasekar M, Thangadurai M, Prabhu PR, Kaliraj P, Ramaswamy K. Immune response studies with Wuchereria bancrofti vespid allergen homologue (WbVAH) in human lymphatic filariasis. Parasitol Res. 2007;101:981-988.

20. Hawdon JM, Jones BF, Hoffman DR, Hotez PJ. Cloning and characterization of Ancylostoma secreted protein. A novel protein associated with the transition to parasitism by infective hookworm larvae. J Biol Chem. 1996;271:6672-6678. 
21. Bin Z, Hawdon J, Qiang S, et al. Ancylostoma secreted protein 1 (ASP-1homologues in human hookworms. Mol Biochem Parasitol. 1999;98:143-149.

22. Daub J, Loukas A, Pritchard D, Blaxter ML. A survey of genes expressed in adults of the human hookworm Necator americanus. Parasitology. 2000;120:171-184

23. Tawe W, Pearlman E, Unnasch TR, Lustigman S. Angiogenic activity of Onchocerca volvulus recombinant proteins similar to vespid venom antigen 5. Mol Biochem Parasitol. 2000;109:91-99.

24. Liu MA. DNA vaccines: an historical perspective and view to the future. Immunol Rev. 2011;239:62-84.

25. Abhilash S, Gajalakshmi D, Ramaswamy K. Multivalent vaccine for lymphatic filariasis. Procedia Vaccinol 2010;3:12-18.

26. Abraham D, Grieve RB, Mika-Grieve M, Seibert BP. Active and passive immunization of mice against larval Dirofilaria immitis. J Parasitol. 1988;74:275-282.

27. Weil G, Li BW, Liftis F, Chandrasekhar R. Brugia malayi: Antibody responses to larval antigens in infected and immunized jirds. Exp Parasitol. 1992;74:315-323.

28. Dabir S, Dabir P, Goswami K, Reddy MV. Prophylactic evaluation of recombinant extracellular superoxide dismutase of Brugia malayi in jird model. Vaccine. 2008;26:3705-3710.

29. Maizels RM, Blaxter ML, Scott AL. Immunological genomics of Brugia malayi: filarial genes implicated in immune evasion and protective immunity. Parasite Immunol. 2001;23:327-344.

30. Chen J, Hu X, He S, et al. Expression and immune response analysis of Schistosoma japonicum VAL-1, a homologue of vespid venom allergens. Parasitol Res. 2010;106:1413-1418.

31. Anand SB, Kodumudi KN, Reddy MV, Kaliraj P. A combination of two Brugia malayi filarial vaccine candidate antigens (BmALT-2 and $\mathrm{BmVAH}$ ) enhances immune responses and protection in jirds. J Helminthol. 2011;4:1-11.
32. Visser A, Van Zeveren AM, Meyvis Y, et al. Gender-enriched transcription of activation associated secreted proteins in Ostertagia ostertagi. Int J Parasitol. 2008;38:455-465.

33. Bennuru S, Semnani R, Meng Z, Ribeiro JM, Veenstra TD, Nutman TB. Brugia malayi excreted/secreted proteins at the host/parasite interface: stage- and gender-specific proteomic profiling. PLoS Negl Trop Dis. 2009;3:e410.

34. Gray CA, Lawrence RA. A role for antibody and Fc receptor in the clearance of Brugia malayi microfilariae. Eur J Immunol. 2002;32: 1114-1120.

35. Chandrashekar R, Rao UR, Subrahmanyam D. Antibody-mediated cytotoxic effects in vitro and in vivo of rat cells on infective larvae of Brugia malayi. Int J Parasitol. 1990;20:725-730.

36. Akiyama Y, Lubeck MD, Steplewski Z, Koprowski H. Induction of mouse IgG2a- and IgG3-dependent cellular cytotoxicity in human monocytic cells (U937) by immune interferon. Cancer Res. 1984;44: 5127-5131.

37. Leu RW, Robinson CJ, Wiggins JA, Shannon BJ, Rummage JA, Horn MJ. Photometric assays for FcRI-dependent binding, phagocytosis, and antibody-dependent cellular cytotoxicity mediated by monomeric IgG gamma $2 \mathrm{a}$ in murine peritoneal macrophages. J Immunol Methods. 1988;113:269-278.

38. Mohr E, Cunningham AF, Toellner KM, et al. IFN-\{gamma $\}$ produced by CD8 T cells induces T-bet-dependent and -independent class switching in B cells in responses to alum-precipitated protein vaccine. Proc Natl Acad Sci U S A. 2010;107:17292-17297.
Research and Reports in Tropical Medicine

\section{Publish your work in this journal}

Research and Reports in Tropical Medicine is an international, peerreviewed, open access journal publishing original research, case reports, editorials, reviews and commentaries on all areas of tropical medicine, including: Diseases and medicine in tropical regions; Entomology; Epidemiology; Health economics issues; Infectious disease; Laboratory

\section{Dovepress}

science and new technology in tropical medicine; Parasitology; Public health medicine/health care policy in tropical regions; and Microbiology. The manuscript management system is completely online and includes a very quick and fair peer-review system. Visit http://www.dovepress com/testimonials.php to read real quotes from published authors. 